\title{
The determination of the critical parameters of a gasoline petroleum fractionSB-180 Trinity- Anastasievskoe oil
}

\author{
Mikhail Chebotarev ${ }^{1}$, and Pavel Kharchenko, ${ }^{1, *}$ \\ ${ }^{1}$ Department of machine repair and materials science, Kuban SAU, Russia
}

\begin{abstract}
Annotation. The article determines the critical parameters of the oil fraction-temperature, pressure and density. The experimental results are compared with the calculated results, the calculation method closest to the experimental results is chosen, the calculation error is estimated. The main results and conclusions are presented. The use of the theory of thermodynamic similarity in the method of density calculation and DNP required first of all knowledge of the parameters of the state at the critical point. The accuracy of the calculation of critical parameters affects the reliability of the results.
\end{abstract}

\section{Temperature}

Currently, the following formulas are used to calculate the critical temperatures of petroleum products:

1. Filippov Formula

$$
T_{C R}=0,494 \cdot\left(T_{0}+\frac{\rho_{0}}{\gamma}\right)
$$

where $\gamma$ is the value of the temperature density correction;

$\mathrm{To}=293.15 \mathrm{~K}$-for petroleum products with $\mathrm{Tz}<20^{\circ} \mathrm{C}$;

$\mathrm{To}=343.15 \mathrm{~K}$-for petroleum products with $\mathrm{Tz}>20^{\circ} \mathrm{C}$.

The value $\gamma$ is determined by the formula

$$
\gamma=\frac{1}{1,706-\frac{43,65}{M \cdot R_{E}}}
$$

where RE is the specific refraction by Eikmann, is determined by the equation

\footnotetext{
*Corresponding author: 1960324@mail.ru
} 


$$
R_{E}=\frac{\left(n_{D}^{t_{0}}\right)^{2}-1}{n_{D}^{t_{0}}+0,4} \cdot \frac{1}{\rho_{4}^{20}}
$$

where $n_{D}^{t_{0}}$ is the refractive index;

$\rho_{4}^{20}$-relative density;

2. Nokei Formula

$$
\lg \left(1,8 \mathrm{~T}_{\mathrm{CR}}\right)=1,28053+0,2985 \lg \rho_{4}^{20}+0,62164 \lg (1,8 \mathrm{~T}),
$$

where $\rho_{4}^{20}$ is the relative density of the product at $20^{\circ} \mathrm{C}$;

T - AVBP.

3. Rot formula

$$
1,2979\left(\rho_{0}-\rho_{1}\right) T_{C R}^{2}-0,54957\left(\rho_{0} T_{1}-\rho_{1} T_{0}\right) T_{C R}-0,09247\left(\rho_{0} T_{1}^{2}-\rho_{1} T_{0}^{2}\right)=0,
$$

where $\mathrm{T}_{1}-\mathrm{T}_{0}>20$;

$\rho_{1}-\rho_{0}$-densities at appropriate temperatures.

In addition, the nomogram method of the American petroleum Institute API is used to calculate critical temperatures [1]. As the initial data for the nomogram, the density of the product at $\mathrm{t}=20^{\circ} \mathrm{C}$ and $\mathrm{AVBP}$ is used.

We calculated the critical temperatures of the studied products by various methods, and found that the most accurate calculation is given by the Nokei formula. To describe the experimental data, we used the Nokei formula used to calculate the critical temperatures of pure hydrocarbons in the form of

$$
\lg T_{c}=A+B \lg S \cdot v+\operatorname{clg} T_{b}
$$

The relative density of the product at $20^{\circ} \mathrm{C}$ and atmospheric pressure was used as the value $\mathrm{S} * \omega$, and the average volume boiling point of the oil product $(\mathrm{K})$ was used as $\mathrm{Tb}[2,3,4]$.

The coefficients A, B, C obtained during the processing of experimental data by the least squares method have the following form: $A=1,1004405 ; B=0.2498864 ; C=0.652894$.

The obtained coefficient values are close to the coefficients used in the Nokei equation for calculating the critical temperatures of olefin hydrocarbons.

Table 1. The critical temperature of petroleum fractions

\begin{tabular}{|c|c|c|c|c|c|}
\hline \multirow{2}{*}{$\begin{array}{c}\text { Sampling } \\
\text { temperatu } \\
\text { re ,Co }\end{array}$} & \multicolumn{5}{|c|}{ Critical temperature, K } \\
\cline { 2 - 6 } & expert. & API & Filippov & Nogai (4) & Nogai (6) \\
\hline $\begin{array}{c}\text { SB-180 t- } \\
\text { a.n. }\end{array}$ & 583,86 & 575,15 & 599,17 & 577,40 & 582,25 \\
\hline$\delta, \%$ & - & 1,3 & 1,64 & 0,93 & 0,13 \\
\hline
\end{tabular}

The table shows that the values of critical temperatures calculated by the formula (6) are the closest to the experimental ones. The mean square error of calculation according to the formula (1) and the nomogram method is an order of magnitude higher than according to the formula (6).

\section{Pressure}


To calculate the critical pressures of the studied oil fraction we used:

The formula for calculation of critical pressures of the products of cracking:

$$
P_{C R}=0,9414 \cdot 10^{6} \cdot T_{b}^{(-2,017)} \cdot d_{4}^{20},
$$

where $\mathrm{T}_{\mathrm{b}}$ is the average boiling point;

$d_{4}^{20}$-density at $20^{\circ} \mathrm{C}$.

Formula for calculating critical pressures of pure hydrocarbons:

$$
\lg P_{C R}=0,00055308 \frac{T_{S}}{T_{C R}} \cdot \frac{T_{C R}-T_{S}}{\left(\frac{\partial T}{\partial P}\right)_{760}},
$$

where $\mathrm{T}_{\mathrm{CR}}$ is the critical temperature, $\mathrm{K}$;

$\mathrm{T}_{\mathrm{S}}$-boiling point;

$\left(\frac{\partial T}{\partial P}\right)_{760}$-selected for a specific hydrocarbon group.

The formula used for the calculation of critical pressures of jet fuels:

$$
P_{C R}=\left(\mathrm{c} \frac{T_{C R} \cdot \rho_{C R}}{M}-D\right) \cdot 10^{5},
$$

where $\mathrm{T}_{\mathrm{CR}}$ is the critical temperature, $\mathrm{K}$;

$\rho_{\mathrm{CR}}$-critical density, $\mathrm{kg} / \mathrm{m} 3$;

$\mathrm{C}$ and $\mathrm{D}$ are constants.

Expressions such as the Nokia equation:

$$
\lg P_{C R}=A+B \lg \rho_{4}^{20}+\operatorname{clg} T_{A V B P},
$$

Table 2. Critical pressures of oil fraction

\begin{tabular}{|c|c|c|c|c|c|}
\hline \multirow{2}{*}{$\begin{array}{c}\text { Sampling } \\
\text { temperature } \\
\text {,Co }\end{array}$} & \multicolumn{5}{|c|}{ Critical pressures, MPa } \\
\cline { 2 - 6 } & $\begin{array}{c}\mathbf{P}_{\mathbf{C R}} \\
\mathbf{( 7 )}\end{array}$ & $\mathbf{P}_{\mathbf{C R}}(\mathbf{8})$ & $\mathbf{P}_{\mathbf{C R}}$ (9) & $\mathbf{P}_{\mathbf{C R}}$ (10) & $\mathbf{P}_{\mathbf{E X P}}$ \\
\hline SB-180 t-a.n. & 2,920 & 3,008 & 2,456 & 3,128 & 3,140 \\
\hline$\delta, \%$ & 8,96 & 4,25 & 20,17 & 3,6 & - \\
\hline
\end{tabular}

The table shows the calculated values of RCR according to the above formulas and their comparison with the experimental ones. The standard error of calculation is given. The maximum error is obtained when calculating by equation (9), the best result is obtained by using an expression of the form Nokei, where $A=2,11216 ; B=0,796456 ; C=0,235387$.

\section{Density (specific volume)}

For calculation of critical values of density of oil products are used:

Filippov formula for calculation of critical values of oil fraction density:

$$
\rho_{C R}=\left[\frac{\left(\rho^{9}-\rho_{0}^{9}\right) \cdot T R}{P-P_{0} \cdot 1080 M}\right]^{\frac{1}{8}},
$$

where $\rho$ and $\rho_{0}, \mathrm{P}$ And $\mathrm{P}_{0}$-refer to two points of the isotherm; R-universal gas constant;

M-molar mass. 
Formula for calculation of critical values of density of jet fuels and pure hydrocarbons:

$$
\rho_{C R}=\frac{10^{-5} \cdot P_{\mathrm{KP}}+D}{C} \cdot \frac{M}{T_{C R}}
$$

The authors indicate that the error of density calculation for (11) is $\pm 1 \%$, for (12) $\pm 2 \%$.

To calculate the critical values of the density of the oil fraction studied by us an expression of the type of the Nokay equation was used

$$
\lg \rho_{C R}=A+B \lg \rho_{4}^{20}+C \lg T_{A V B P}
$$

The calculation results are given in the table.

Table 3. Result of calculation

\begin{tabular}{|c|c|c|c|}
\hline $\begin{array}{c}\text { The name of the } \\
\text { faction }\end{array}$ & $\mathbf{P}_{\mathrm{EX}} \mathbf{\Gamma} / \mathbf{c m}^{\mathbf{3}}$ & $\mathbf{P}_{\mathbf{R}}(\mathbf{1 3}) \mathbf{g} / \mathbf{c m 3}$ & $\boldsymbol{\omega}, \mathbf{\%}$ \\
\hline SB-180 t-a.n. & 0,2409 & 0,2409 & 0 \\
\hline
\end{tabular}

The mean square error of the calculation was $0.04 \%$. Coefficients of equation (13) $\mathrm{A}=0,04838144 ; \mathrm{B}=0,92861442 ; \mathrm{C}=-0,21308121$.

Thus, using expressions such as the Nokei equation, one can calculate the $\mathrm{T}_{\mathrm{KP}}, \mathrm{P}_{\mathrm{KP}}$ and $\rho_{\mathrm{KP}}$ of the gasoline oil fraction.

The generalized dependence of density on saturation lines in the critical region is presented in Fig. 1.

Table 4 shows the values of the critical parameters of the studied fraction.

Table 4. Critical parameters of the faction

\begin{tabular}{|c|c|c|c|}
\hline $\begin{array}{c}\text { The name of the } \\
\text { faction }\end{array}$ & $\mathbf{T}_{\mathbf{K R}}, \mathbf{K}$ & $\mathbf{P}_{\mathbf{K R}}, \mathbf{M P a}$ & $\mathbf{\rho}_{\mathrm{KR}}, \mathbf{k g} / \mathbf{~ c m 3}$ \\
\hline SB-180 t-a.n. & 583,86 & 3,140 & 240,9 \\
\hline
\end{tabular}

\section{Estimation of the error of pressure measurement}

When calculating the error of the pressure measurement was based on the working formula:

$$
P_{E P}=B-0,0417 \cdot \rho_{X}^{P}+0,981 \cdot P_{M},
$$

where $\mathrm{P}_{\mathrm{EP}}$-experimental pressure $(\mathrm{MPa} * 10-1)$;

$\mathrm{B}$-barometric pressure $(\mathrm{MPa} * 10-1)$;

$0,0417 \rho_{\mathrm{X}}^{\mathrm{P}}$ - correction for pressure associated with the difference in liquid levels in the piezometer and the measuring column of the pressure gauge (bar);

$\mathrm{P}_{\mathrm{M}}$-manometric pressure $(\mathrm{kgf} / \mathrm{cm} 2)$.

The confidence interval of each variable is less than the instrumental error, so to characterize the total error of the measured value $\mathrm{P}$, we use the instrumental errors of all variables:

$$
\Delta P=t(\alpha) \cdot \sqrt{\Delta P_{M}^{2}+\Delta P_{\delta}^{2}+\Delta P_{P}^{2}+\Delta P_{\bar{Z}}^{2}}
$$


where $\triangle \mathrm{PM}$-the error of measurement of the piston gauge $(\mathrm{MO}-\triangle \mathrm{PM}=0.0016 \mathrm{P}$; MP6$\Delta \mathrm{PM}=0.0002 \mathrm{P} ; \mathrm{MP} 60, \mathrm{MP} 600-\Delta \mathrm{PM}=0.0005 \mathrm{P})$.

Accuracy of mercury barometer pressure measurement:

$$
\Delta P_{\delta}=0,0001, P_{\delta}=0,1 \cdot 10^{-4} \text { МПа }
$$

The error introduced by inaccuracy of measurement of heights of a liquid in system:

$$
\Delta P_{\bar{Z}}=0,0002 \mathrm{M \Pi а}
$$

The sensitivity of the membrane separator:

$$
\Delta P_{P}=0,0001 \mathrm{M \Pi а}
$$

Then the total error of the pressure measurement:

$$
\left.\begin{array}{c}
\Delta P=1,1 \cdot \sqrt{\Delta P_{M}^{2}+0,00001^{2}+0,0001^{2}+0,0002^{2}} \\
\text { МО и } M P 6-\Delta P=0,00025 \div 0,00036 \mathrm{MPa} \\
M P 60-\Delta P=0,0004 \div 0,0033 \mathrm{MPa} \\
M P 600-\Delta P=0,0033 \div 0,0320 \mathrm{MPa}
\end{array}\right\}
$$

\section{Estimation of temperature measurement error}

Systematic and random errors were taken into account when estimating the temperature measurement error.

The random error of temperature measurement $\delta \mathrm{t}$ With a model platinum resistance thermometer is calculated in detail in $[5,6,7]$. The systematic error $\Delta$ tpts and the thermostatic error $\Delta \mathrm{tt}$ and is determined from the expression:

$$
\Delta t_{S}=1,1 \cdot \sqrt{\Delta t_{P T S}^{2}+\Delta t_{\mathrm{T}}^{2}}
$$

The authors $[1,8]$ calculated the error $\Delta$ tPTS for a standard measuring circuit at different temperatures. Since our measurement scheme used a similar measuring potentiometric setting, we will use the results $[5,8]$ to determine the error of temperature measurement. The data of temperature measurement errors are presented in table 3.4.

Table 5. Error of measurement of temperature

\begin{tabular}{|c|c|c|c|c|}
\hline \multirow{2}{*}{ Type of error } & \multicolumn{4}{|c|}{ Error $\Delta \mathrm{t} \cdot 10^{3},{ }^{\circ} \mathrm{C}$} \\
\cline { 2 - 5 } & $0{ }^{\circ} \mathrm{C}$ & $100{ }^{\circ} \mathrm{C}$ & $200{ }^{\circ} \mathrm{C}$ & $300{ }^{\circ} \mathrm{C}$ \\
\hline$\Delta \mathrm{t}_{\mathrm{T}}$ & 0,35 & 0,7 & 0,7 & 0,7 \\
\hline$\Delta \mathrm{t}_{\mathrm{PTS}}$ & 4 & 13 & 20 & 21 \\
\hline$\Delta \mathrm{ts}$ & 5,52 & 14,7 & 22.3 & 23,3 \\
\hline
\end{tabular}

\section{Evaluation of measurement uncertainty of specific volume}

The specific volume of the investigated liquid was calculated by the expression: 


$$
G_{t, P}=\frac{V_{0}-V_{n}^{K}-V_{T}^{K}+\Delta V_{t}+\Delta V_{P}}{m_{0}-m_{n}^{K}-m_{T}^{K}-\sum_{0}^{n} m_{i}},
$$

where $\mathrm{V}_{0 \text { - }}$ is the internal volume of the piezometer at $\mathrm{P}=\mathrm{Pat}, \mathrm{t}=20^{\circ} \mathrm{C}$;

$\mathrm{V}_{\mathrm{n}}^{\mathrm{K}}$-volume of capillaries in the zone of variable temperatures;

$\mathrm{V}_{\mathrm{T}}^{\mathrm{K}}$ - volume of capillaries in the thermostatic zone;

$\Delta V_{\mathrm{t}}$-correction for thermal expansion of the piezometer;

$\Delta \mathrm{V}_{\mathrm{P}}$-correction for isothermal expansion of the piezometer;

$\mathrm{m}_{0}, \mathrm{~m}_{\mathrm{n}}^{\mathrm{K}}, \mathrm{m}_{\mathrm{T}}^{\mathrm{K}}$-masses of liquid in corresponding volumes;

$\sum_{0}^{n} m_{i}$ is the mass of the liquid released from the piezometer.

We find in this formula the corresponding coefficients of influence of each term separately for the numerator and denominator:

$$
\begin{gathered}
\Delta V=1,1 \cdot \sqrt{W_{V_{0}}^{2} \cdot \theta_{V_{0}}^{2}+W_{V_{n}^{K}}^{2} \cdot \theta_{V_{n}^{K}}^{2}+W_{V_{T}^{K}}^{2} \cdot \theta_{V_{T}^{K}}^{2}+W_{\Delta V_{t}}^{2} \cdot \theta_{\Delta V_{t}}^{2}+W_{\Delta V_{P}}^{2} \cdot \theta_{\Delta V_{P}}^{2}}, \\
\Delta m=1,1 \cdot \sqrt{W_{m_{0}}^{2} \cdot \theta_{m_{0}}^{2}+W_{m_{n}^{K}}^{2} \cdot \theta_{m_{n}^{K}}^{2}+W_{m_{T}^{K}}^{2} \cdot \theta_{m_{T}^{K}}^{2}+W_{\Sigma m_{i}}^{2} \cdot \theta_{\Sigma m_{i}}^{2}}
\end{gathered}
$$

Formulas (18) and (19) can be written in the form:

$$
\begin{gathered}
\Delta V=1,1 \cdot \sqrt{1 \cdot \theta_{V_{0}}^{2}+1 \cdot \theta_{V_{n}^{K}}^{2}+1 \cdot \theta_{V_{T}^{K}}^{2}+1 \cdot \theta_{\Delta V_{t}}^{2}+1 \cdot \theta_{\Delta V_{P}}^{2}}, \\
\Delta m=1,1 \cdot \sqrt{1 \cdot \theta_{m_{0}}^{2}+1 \cdot \theta_{m_{n}^{K}}^{2}+1 \cdot \theta_{m_{T}^{K}}^{2}+1 \cdot \theta_{\Sigma m_{i}}^{2}}
\end{gathered}
$$

The confidence limit of systematic error of measurement of specific volume can be calculated by the formula:

$$
\frac{\theta}{v}=1,1 \cdot \sqrt{\frac{\left(\frac{\partial v}{\partial V}\right)^{2} \cdot \Delta V^{2}}{v^{2}}+\frac{\left(\frac{\partial v}{\partial m}\right)^{2} \cdot \Delta m^{2}}{v^{2}}+\frac{\left(\frac{\partial v}{\partial P}\right)^{2} \cdot \Delta P^{2}}{v^{2}}+\frac{\left(\frac{\partial v}{\partial T}\right)^{2} \cdot \Delta T^{2}}{v^{2}}}
$$

To estimate the random error, data on the measurement of specific volumes of water at six isotherms were used. The obtained values were compared with the literature data $[8,9]$. As an approximate estimate of the random error of our experimental data we can take the total for all isotherms estimate of the standard deviation of the measured specific volumes compared with the literature data:

$$
\frac{S_{\bar{X}}}{v}=0,018 \%
$$

As an example, we give an estimate of the measurement error of the specific volume for the fraction $\mathrm{NK}-180^{\circ} \mathrm{C}$ of the Troitsko-anastasievskaya oil at the following parameters:

$$
t=200^{\circ} \mathrm{C}, P=2 \mathrm{MPa}
$$

The systematic error in determining the volume of the piezometer and capillaries are equal respectively: 


$$
\theta_{V_{0}}=0,011 \mathrm{sm}^{3} ; \theta_{V_{n}^{K}}=0,002 \mathrm{sm}^{3} ; \theta_{V_{T}^{K}}=0,008 \mathrm{sm}^{3}
$$

The correction for the isothermal expansion of the piezometer at $\mathrm{P}=2 \mathrm{MPa}$ is 0.006 $\mathrm{cm} 3$ and is made with an error of $1 \%$ (data error $\delta$ and $\mathrm{E}$ for steel X18H10T):

$$
\theta_{\Delta V_{P}}=0,00006 \mathrm{sm}^{3}
$$

The error of correction for the thermal expansion of the piezometer can be found from the approximate dependence:

$$
\theta=2 \cdot S_{\Delta V}
$$

where $\mathrm{S} \Delta \mathrm{V}$ is an estimate of the standard deviation of the experimental values $\Delta \mathrm{V}$ from the approximating curve.

For temperature range $20 \div 300{ }^{\circ} \mathrm{C}$ :

$$
\theta=2,8 \%
$$

The correction for the thermal expansion of the piezometer at $200{ }^{\circ} \mathrm{C}$ is $1,195 \mathrm{~cm} 3$, then:

$$
\theta_{V_{t}}=0,039 \mathrm{sm}^{3}
$$

Systematic errors in determining the mass of the test substance in the piezometer and capillaries coincide with the error in determining the corresponding volumes.

When assessing the mass error of the liquid merged from the piezometer, the requirements of GOST 19491-74, the error from the nonequilibrium of the weights, the mass error of the weights were taken into account.

Losses due to evaporation lay in the range of 0.004-0.006 $\mathrm{g}$ during the measurement on the isotherm, which was 14-16 hours.

As an absolute error in determining the mass of the evaporated liquid, a double variation of the weights ADV-200 - 0.0002 was adopted. the Correction was distributed proportionally to the time from the first drain to the last point on this isotherm.

The confidence limit of the systematic error of measuring the mass of the liquid at discharge was:

$$
\theta_{\bar{Z} m_{i}}=0,001 \mathrm{~g}
$$

Substituting the obtained values into formulas (13) and (19) we obtain:

$$
\Delta V=0,066 \mathrm{sm}^{3} ; \Delta m=0,015 \mathrm{sm}^{3}
$$

The relative systematic error in determining the volume is:

$$
\frac{\left(\frac{\partial v}{\partial V}\right) \cdot \Delta V}{v}=0,19 \cdot 10^{-3}
$$

At an isotherm of $200{ }^{\circ} \mathrm{C}$ at $\mathrm{P}=2 \mathrm{MPa}, 60.2 \mathrm{~g}$ of the substance remained in the piezometer, then:

$$
\frac{\left(\frac{\partial v}{\partial m}\right) \cdot \Delta m}{v}=0,79 \cdot 10^{-4}
$$


Attribution errors were determined using large scale graphs of isotherms and isobars:

$$
\begin{aligned}
& \frac{1}{v} \cdot\left(\frac{\partial v}{\partial P}\right)_{T} \cdot \Delta P \approx \frac{1}{v} \cdot \frac{v-v_{1}}{P-P_{1}} \cdot \Delta P=0,19 \cdot 10^{-5}, \\
& \frac{1}{v} \cdot\left(\frac{\partial v}{\partial T}\right)_{P} \cdot \Delta T \approx \frac{1}{v} \cdot \frac{v-v_{1}}{T-T_{1}} \cdot \Delta T=0,41 \cdot 10^{-4} .
\end{aligned}
$$

The systematic error in determining the specific volumes of the substance at the filling parameters was determined by the error in determining the specific volumes at the plant by the method of hydrostatic weighing and was accepted according to [3]:

$$
\frac{\Delta v}{v}=0,17 \cdot 10^{-3}
$$

Substituting the obtained values into the formula (15), we obtain:

$$
\frac{\theta_{v}}{v}=0,029 \%
$$

We define the boundary of the confidence interval of the random error by the formula (4). At $\alpha=0.95$ and the number of degrees of freedom $K=10$, the percentage point of the student's distribution $\operatorname{tg}=2.23$, whence $\Psi=0.04 \%$.

The standard deviation of the systematic error :

$$
\frac{S_{v}}{v}=\sqrt{\frac{1}{3} \cdot 0,69 \cdot 10^{-3}=0,015 \%}
$$

Then the standard deviation of the total error:

$$
\frac{S_{\Sigma}}{v}=\sqrt{0,018^{2}+0,015^{2}=0,023 \%}
$$

The value of $\mathrm{t} \Sigma$ is determined :

$$
t_{\Sigma}=\frac{0,029+0,04}{0,018+0,015}=2,09
$$

Then the confidence limit of the total error of the measurement result is determined:

$$
\Delta=0,023 \cdot 2,09=0,048 \%
$$

Similar calculations were performed for other areas of state parameters. For the studied fraction, the confidence limit of the total error of the measurement result lies in the range of $0.03 \div 0.1 \%$. At temperatures close to critical, the influence of the reference errors and the error associated with the introduction of an amendment to the thermal expansion of the piezometer increases.

In the two-phase region, the confidence limit of the total error increases and lies within $0.08 \div 0.15 \%$. This is due to a sharp increase in this area of the error of the pressure reference and the error of determining the mass of the substance in the piezometer [10]. 


\section{Main results and conclusions}

1. It is established that experimental P-and-t data obtained near the boundary curve, in the two-phase and critical regions are few. The calculation methods rely mainly on experimental P-and-t data of individual hydrocarbons and are not reliable enough.

2. The choice of the measurement method is justified, an experimental setup for the study of the density and pressure of saturated vapors of petroleum products is developed and created. The error of the results is $0.03-0.1 \%$ for density and $0.05-0.5 \%$ for saturated vapor pressure. The reliability of the error estimation is confirmed by measurements of density and pressure of saturated water vapors.

3. Formulas are obtained to calculate the density and pressure on the saturation lines, as well as the pressure of saturated vapors in the two-phase region.

4. As a result of the analysis of experimental data in the critical area, the conclusion is made about the identity of critical and pseudocritical parameters of the studied oil fractions.

5. Dependence for calculation of critical parameters $\left(T_{\mathrm{KP}}, \mathrm{P}_{\mathrm{KP}}, \rho_{\mathrm{KP}}\right)$ of gasoline fractions using density at $\mathrm{t}=20^{\circ} \mathrm{C}$ and average volume boiling point is found.

6 . The validity of the method of studying isotherms in the two - phase region for the study of $\mathrm{P}-\mathrm{u}$-t dependence of multicomponent hydrocarbon systems (oil fractions) is proved.

\section{References}

1. P. Kharchenko, Proceedings of the Kuban State Agrarian University, 39, 140-142 (2012)

2. P. Kharchenko, V. Timofeev, Polythematic network electronic scientific journal of the Kuban State Agrarian University, 98, 528-543 (2014)

3. V. Khristichenko, N. Blosinsky, Proceedings of the Kuban State Agrarian University, 37, 238-242 (2012)

4. V. Timofeev, Polythematic network electronic scientific journal of the Kuban State Agrarian University, 103, 973-982 (2014)

5. D. Chizhov, Polythematic network electronic scientific journal of the Kuban State Agrarian University, 106, 1000-1012 (2015)

6. V. Timofeev, D. Chizhov, Polythematic network electronic scientific journal of the Kuban State Agrarian University, 107, 779-792 (2015)

7. B. Valverde, Grop Protest, 62, 16-22 (2014)

8. P. Tehranchian, Weed Science, 63(3), $561-568$ (2015)

9. R. Pedroso, Sci, 72 (9), 561 - 568 (2016)

10. R. Gavaerts, Royal Botanic Gardens, Kew, 726 (2014) 